\title{
La utilidad del feminismo. Empoderamiento y visibilización de la violencia urbana en las mujeres jóvenes
}

\author{
The Utility of Feminism. Empowerment and Visibility of Urban \\ Violence in Young Women
}

\author{
Lionel S. Delgado ${ }^{1}$ \\ Fecha de recepción: 29-03-2018 - Fecha de aceptación: 13-06-2018 \\ Hábitat y Sociedad (ISSN 2173-125X), n. ${ }^{\circ}$ 11, noviembre de 2018, pp. 131-148. \\ http://dx.doi.org/10.12795/HabitatySociedad.2018.i11.08
}

\begin{abstract}
Social innovation in urban policies is an un-trodden path, especially in the gender urbanism issue. Feminism, as an empowering discourse, has a great importance for urban policies due to its potential in detecting and assessing the episodes of urban violence suffered by women. To support this idea, twenty-one in-depth interviews were conducted with youth women (18-26 years) divided by levels of commitment to feminism. The differences of the interviewees when identifying and explaining the dynamics of harassment and violence suffered allow us to analyze how the presence/absence of feminist discourse intervenes in the way of living aggressions in the urban context. In each group, a certain level of critical awareness is combined with the prevailing need to operate as urban agents, which gives rise to different survival strategies and risk management.
\end{abstract}

\section{Key words}

Empowerment; Feminism; Urban Policies; Social Innovation; Urban Harassment

\begin{abstract}
Resumen
La innovación social en las políticas urbanas es un campo poco transitado, especialmente en relación a la ciudad desde la perspectiva de género. El feminismo, en tanto discurso empoderador, guarda una gran importancia para las políticas urbanas debido a su potencial en la detección y valoración de las dinámicas de violencia urbana sufrida por mujeres. Para apoyar esta idea, se han realizado entrevistas en profundidad a mujeres jóvenes (18-26 años) divididas por niveles de compromiso con el feminismo. Las diferencias que presentan las entrevistadas a la hora de identificar y explicar las dinámicas de acoso y violencia sufridas permiten analizar cómo la presencia/ausencia de discurso feminista interviene en la forma de vivir las agresiones en el contexto urbano. En cada grupo se conjuga un determinado nivel de conciencia crítica con la necesidad imperante de operar como agentes urbanos, lo cual da pie a distintas estrategias de supervivencia y gestión del riesgo.
\end{abstract}

\section{Palabras clave}

Empoderamiento; Feminismo; Políticas Urbanas; Innovación social; Acoso callejero

1 Doctorando de Sociología en la Universidad de Barcelona; investigador en temas de Sociología Urbana, Movilidad Juvenil, Urbanismo con Perspectiva de Género, Feminismo y Estudios sobre Masculinidades. E-mail: lionel.s.delgado@gmail.com. 


\section{Introducción}

Decía Naila Kabeer (1999) que es importante ligar los logros del feminismo a las prioridades de las agendas institucionales para conseguir mejores sinergias entre las administraciones y el movimiento feminista (cfr. p. 435). Esta no es una cuestión baladí: en un contexto de austeridad presupuestaria en las políticas sociales, defender el interés del feminismo defendiendo su utilidad para las políticas públicas resulta valioso para una mayor difusión de los valores de igualdad de género en la praxis política. Asimismo, centrar el discurso en las ventajas que el enfoque feminista aporta a la sociedad permite romper con los estereotipos que ven en el feminismo una lucha sectorial de "suma cero" cuyos avances suponen un retroceso para otros grupos (cfr. ob. cit., p. 436). Asimismo, "hablar su idioma" en términos de eficacia, eficiencia y mejora de las políticas públicas puede ser una buena herramienta para promover que gobierno y administraciones integren nuevos planteamientos de igualdad.

Si bien a lo largo de las últimas décadas se ha conseguido implantar un enfoque de género en las políticas públicas (gender-oriented policy) en varias áreas, en las políticas urbanas aún queda mucho por recorrer. El enfoque de género en las políticas urbanas es muy reciente y, aunque se llevan planteando debates sobre este tema desde los años setenta, no adquiere la legitimidad de un tema digno hasta décadas después debido al carácter masculinizado y tradicional del campo académico del urbanismo y la arquitectura (Durán Heras, 2017). ${ }^{2}$ No obstante, en los últimos años se dará una verdadera efervescencia académica en relación al urbanismo con perspectiva de género, con abordajes desde diversas disciplinas y estudios de distintos ámbitos que revelan la potencialidad y riqueza de un tipo de enfoque urbano lleno de posibilidades. ${ }^{3}$

A su vez, el desarrollo del enfoque de la innovación social y la gobernanza urbana, haciendo hincapié en las iniciativas que surgen desde abajo para afrontar necesidades no satisfechas de los ciudadanos (cfr. Pradel Miquel y García Cabeza, 2018, p. 21), permite una lectura por la que el feminismo puede entenderse como un motor de procesos de innovación social al intervenir en la forma en la que la ciudadanía se relaciona con la ciudad. En tanto discurso que permite resignificar realidades urbanas, visibilizar desigualdades y defender un modelo de ciudad inclusiva y disfrutable para todas y todos (Muxí, Casanovas, Ciocoletto et al., 2011), la difusión de una perspectiva feminista es un elemento valioso para las políticas urbanas, entendiendo por tales algo más que políticas locales, políticas urbanísticas y políticas públicas (cfr. Blanco y Subirats, 2012, p. 18); una articulación multinivel de actores, institucionales y no institucionales, volcados hacia la solución de distintos problemas en las ciudades.

Para estudiar la potencialidad del feminismo como elemento de innovación social se ha realizado un trabajo de campo en la ciudad de Zaragoza investigando la influencia del acoso callejero en la percepción urbana de mujeres jóvenes (Delgado y Aguerri, 2018). En este artículo, las entrevistas en profundidad realizadas a mujeres jóvenes (1826 años) se han dividido por niveles de cercanía al feminismo para un análisis de discurso que permita ver la relación entre la concienciación feminista y los procesos de empoderamiento relacionados con la percepción y valoración de situaciones de agresión en contextos urbanos.

En el presente artículo nos centramos en el perfil de la mujer joven. Se opta por un perfil concreto porque resulta incorrecto hablar 
de "la mujer" en general, ya que pasa por alto que las interacciones de la mujer con el entorno urbano están estructuradas por diversos ejes de desigualdad; es necesario centrarnos en un colectivo concreto para analizar el cruce de los ejes de desigualdad mencionados, siendo especialmente cuidadosos con las distintas dimensiones de la opresión, como plantea el enfoque de la interseccionalidad (McCall, 2005). Nos centramos en las mujeres jóvenes porque existe un vacío bibliográfico en los estudios sobre la vida urbana de la juventud con perspectiva de género (Rodó-de-Zárate, 2011).

Para ello, el artículo se estructura en tres partes. En la primera se desarrollará el aparato teórico donde se presentarán los elementos que hilarán la lectura de las entrevistas: se argumentará sobre la importancia de la innovación social para las políticas urbanas y sobre cómo el empoderamiento femenino resulta un elemento importante a tener en cuenta. En la segunda parte se desarrollará la metodología utilizada, así como las hipótesis planteadas. Finalmente, en la tercera parte se expondrán los resultados. Se cerrará con las conclusiones que repasarán qué hipótesis se han podido ver apoyadas por los resultados y cuáles ha habido que rechazar o matizar.

\section{Innovación social y exclusión urbana de la mujer}

La innovación social supone la puesta en práctica de nuevas aproximaciones sociales a los problemas de exclusión (Pradel Miquel y García Cabeza, 2018). Una suerte de inteligencia pragmática ciudadana que organiza recursos para una práctica empoderadora. Según la definición de Moulaert (citado en González, Moulaert y Martinelli, 2010), la innovación social:

(...) se produce cuando la movilización de las fuerzas sociales e institucionales tiene éxito en el logro de la satisfacción de las necesidades humanas previamente enajenadas, la potenciación relativa de grupos sociales previamente excluidos o silenciados a través de la creación de nuevas 'capacidades' y, en última instancia, cambios en las relaciones sociales y de poder existentes tendentes hacia un sistema de gobernanza más inclusiva y democrática (p. 54).

Como se ve, vuelve a relacionarse innovación social con la satisfacción de necesidades no resueltas ni por el Estado ni por el Mercado. La organización social permite un proceso de empoderamiento ciudadano (Mulaert, McCallum, Mehnood et al., 2013) que no sólo hace encontrar las distintas necesidades insatisfechas en un proceso común de empoderamiento colectivo, sino que desarrolla una conciencia común sobre la naturaleza de los problemas y las formas de afrontarlos.

Así, innovación social y empoderamiento se encuentran íntimamente ligados: los episodios de innovación social abren (y son alimentados por) dinámicas de empoderamiento que dotan a la ciudadanía de herramientas para dar respuesta a las necesidades no resueltas. Es un proceso de retroalimentación donde causa y consecuencia son intercambiadas de manera dinámica.

No obstante, al ligar innovación social con respuestas a diversos procesos de exclusión (cfr. Pradel Miquel y García Cabeza, 2018, p. 15) no sólo deberíamos pensar en el desempleo, los problemas de vivienda, las carencias de recursos básicos o la gestión de equipamientos públicos. 
La exclusión urbana es también un fenómeno fundamental que afecta a grupos sociales específicos definiendo su vida social. En las distintas formas de exclusión urbana, la situación que vive la mujer en la ciudad tiene unas características muy específicas y ha necesitado un enfoque propio en el que se aborde la realidad urbana con perspectiva de género. Este enfoque ha resultado muy productivo en las últimas décadas (Sandercock y Forsyth, 1992; Coffey, 1995; Greed, 1996; Bridge y Watson, 2000; Ortiz i Guitart, 2007) echando luz sobre la especificidad de género de la experiencia de la ciudad.

Se trata de abordar la situación de la mujer, no sólo desde la desigualdad basada en la cantidad de recursos poseídos (como pasa con las demandas económicas, habitacionales o de recursos básicos) sino en lo que la situación permite a las personas hacer o ser. Se trata de acercarnos más al enfoque de las capacidades (Capability Approach) desarrollado por Amartya Sen $(1984 ; 1985)$ por el cual la pregunta gira en torno a las herramientas que adquieren las personas para alcanzar actos o estados valiosos. La innovación social, en este sentido, podría enfocarse desde la producción de procesos que repercutan en el desarrollo de capacidades ciudadanas para cambiar las situaciones de desigualdad (Chiappero-Martinetti, Houghton Budd y Ziegler, 2017). En este caso, podría entenderse el problema de la mujer y la ciudad como un problema de capacidades: las habilidades de la mujer para poder disfrutar de la ciudad de una manera igualitaria se ven mermados por diversos factores (objetivos y subjetivos).

La vida urbana puede analizarse desde tres indicadores (Cresswell, 2010): desplazamientos físicos, representaciones y prácticas. Los más usuales son los análisis sobre desplazamientos (Díaz, 1989; MirallesGuasch, 1998; Olmo Sánchez y Maeso González, 2012), sin embargo, los movimientos están ligados también a representaciones y prácticas cotidianas. Los problemas de la mujer no son sólo de dificultades para desplazarse (responsabilidades ligadas al mercado laboral y al cuidado de personas con diversos niveles de dependencia, menor acceso a un coche privado, etc.) (Sánchez de Madariaga, 2004), sino que la percepción y las prácticas cotidianas también presentan retos: la forma en la que la mujer percibe la ciudad está atravesada por el miedo y la inseguridad (MacMillan, Nierobisz y Welsh, 2000; Pain, 2001; Fairchild y Rudman, 2008; Añover López, 2012), fruto de los casos de acoso callejero vividos en primera persona o aprendidos a través de terceros (amigos, familiares o medios de comunicación). Esto afecta a la capacidad de reapropiación de los espacios de la ciudad y, por lo tanto, a las prácticas de acción-transformación que la mujer puede llevar a cabo.

Estos elementos de acción y vinculación afectiva son la clave para poder vincularse afectivamente y apropiarse de ellos (Vidal Moranta y Pol Urrutia, 2005). Sin embargo, su aparición no es espontánea: en una situación de desigualdad social, la vinculación afectiva y la accióntransformación se darán unicamente a través de un proceso de empoderamiento, entendido como el proceso de incrementar el poder personal, interpersonal o político para que los individuos, familias o comunidades puedan ponerse manos a la obra para mejorar sus situaciones (Gutiérrez, 1995). Este empoderamiento permitirá que la mujer rompa con una visión victimista de sí misma y genere procesos de innovación social que intenten dar respuesta a una necesidad de inclusión urbana sin resolver.

Este proceso de empoderamiento tiene un factor cognitivo importante: según Lorraine M. Gutiérrez (1995), la conciencia crítica, 
necesaria para el proceso de empoderamiento, consta de tres procesos psicológicos: el Group Identification (identificación de áreas de experiencias comunes que construyen una noción de grupo), el Group Consciousness (comprensión de las diferencias de poder y status sociales junto a un sentimiento de privación relativa que lleva al descontento social) y el Self and Collective Efficacy (creencia en que uno es capaz de realizar cambios deseables en la propia vida). Son tres componentes cognitivos de la conciencia crítica que se pueden articular mutua o independientemente.

Entre las condiciones objetivas y las prácticas sociales no siempre hay una relación directa, por eso es necesario la atención sobre los factores cognitivos, los cuales cumplen el papel de creencias subjetivas que operan como mediadoras (Alexander y Welzel, 2011) y que afectan a la valoración de determinadas prácticas. En resumidas cuentas, la legitimidad percibida y la deseabilidad de determinadas realidades son las que estimulan o frenan los procesos de empoderamiento: el nivel de contestación no es comprensible sin incluir en el análisis la forma en la que las mujeres valoran su situación y la deseabilidad del cambio. Cualquier proceso de empoderamiento estará ligado a creencias subjetivas, tanto a aquellas que hacen posible el empoderamiento como a aquellas que surgen a partir del propio empoderamiento.

La mediación de determinados filtros cognitivos será, por ejemplo, la que permita que se visibilicen las dinámicas de desigualdad estructuctural, muchas veces naturalizadas o normalizadas a través de una violencia simbólica capaz de "hacer ver y de hacer creer, de confirmar o de transformar la visión del mundo" (Bourdieu, 2000, p. 71). Esta violencia simbólica actúa sobre la capacidad de la víctima para identificar una violencia como tal, reforzando la situación que la origina. Una agresión puede ser normalizada a través de varios mecanismos cognitivos, lo que influirá en la posibilidad de identificar determinadas realidades como injustas. Así, el filtro cognitivo tiene una importancia clara: permite la identificación de las situaciones de violencia como ilegítimas, lo que facilitaría la apertura de procesos de empoderamiento y episodios de innovación social.

Por lo tanto, resulta fundamental para las políticas urbanas estudiar las creencias que intervienen en la forma en que la mujer percibe y se relaciona con el entorno urbano. Con este enfoque también se evita la victimización que plantea a la mujer como un receptáculo vacío de relaciones de poder: las mujeres son agentes urbanos que, si bien tienen que lidiar con un entorno agresivo, no se ven inhabilitadas como ciudadanas sino que son capaces de adaptarse y de poner en marcha estrategias de supervivencia y resistencia.

\section{Metodología e hipótesis}

La investigación opta por un estudio cualitativo al valorar que este tipo de aproximación consigue indagar más profundamente en los hechos "en los que los seres humanos se implican e interesan, evalúan y experimentan directamente" (Balcázar Nava, González-Arratia, Gurrola Peña et al., 2006, p. 23). Al ser adecuada para la investigación discursiva, la aproximación principal se basa en las entrevistas en profundidad semiestructuradas al permitir abrir las puertas a la vida cotidiana de la persona entrevistada y construye un canal de comunicación donde afloran recuerdos, deseos y creencias. ${ }^{4}$
4 Merece apuntar la atención prestada al proceso de entrevistas debido a ser un hombre el entrevistador. En este caso, las relaciones de poder pueden intervenir de manera significativa, por lo que el proceso debe ser muy cuidadoso. Se opta por la forma semi-estructurada de la entrevista por ofrecer una libertad considerable para facilitar la expresión emocional. Los silencios o frases sin terminar se entenderán como parte del ritmo que marcaba cada entrevistada. El papel del entrevistador se midió con cuidado, teniendo especial tacto con la comunicación corporal, la actitud positiva y la asertividad para fortalecer la confianza. Sabiendo que la entrevista podía esconder relaciones de poder inconscientes, se intentaron mitigar los efectos optando por la pasividad del entrevistador respecto al entrevistado. 
La selección de las entrevistadas se realizó a través de un muestreo secuencial conceptualmente construido (Glaser y Strauss, 1967; Miles y Huberman, 1994), teniendo por objetivo la saturación del discurso (Ibañez, 1997). En este caso, las entrevistas se realizaron a mujeres de nacionalidad españolas de entre 18 y 26 años residentes en la ciudad de Zaragoza, una ciudad de tamaño medio (697.895 habitantes). ${ }^{5}$ Los límites convencionales de la etapa de la juventud suele encontrarse entre los 15 y 29 (Vinuesa Angulo, 2000). En la presente investigación, la edad mínima estudiada se cambia por los 18 años, momento en el que la escuela deja de ser el espacio de encuentro e intercambio cultural para pasar a ocupar ese lugar la ciudad. La plaza, el bar, el lugar de trabajo o la universidad pasan a ser los enclaves de identidad de la juventud. Por otra parte, en lugar de 29 años se opta por los 26 ya que la categorización típica quincenal agrupa un colectivo muy heterogéneo que vive experiencias muy diversas. Se apuesta por reducir la edad superior para garantizar una mejor delimitación experiencial en el estudio. La homogeneidad relativa del grupo estudiado permite mantener fija la unidad experiencial mientras la mirada se centra en la relación entre grado de concienciación y percepción de agresiones.

La apuesta por estudiar mujeres españolas se basa, por una parte, en un intento de controlar las variables que podrían intervenir en el fenómeno estudiado: la tradición de estudios de la interseccionalidad ha demostrado que los ejes de edad, clase, raza y etnia fragmentan la experiencia de las mujeres. Por ello, frente al peligro de mezclar distintos ejes y hacer menos claro el análisis (cfr. McCall, 2005, p. 1787), se opta por controlar las variables de raza, etnia y país de nacimiento en la muestra, aun cuando eso limite la generalización de las conclusiones.

Pretender abordar la universalidad de la experiencia de la agresión urbana de la mujer joven sobrepasa con creces las necesarias limitaciones de un artículo como el presente. Para un estudio de vivencias específicas nos remitimos a otros trabajos según interesen la relación entre ciudad y mujeres migrantes (Pain, 2001; van Lieshout y Aarts, 2008; Ehrkamp, 2013; Sime, 2017), ciudad y jóvenes lesbianas (Rodó-de-Zárate, 2015), personas trans (Doan, 2007), trabajadoras nocturnas (Ortiz Escalante, 2017), mujeres en el entorno rural (Panelli, 2005) o mujeres sin hogar (Casey, Goudie y Reeve, 2008).

Por otra parte, la apuesta por mujeres jóvenes españolas intenta rellenar un vacío en la literatura científica, al ser escasas las contribuciones centradas en mujeres españolas (Rodó-de-Zárate, 2011). No obstante, siendo conscientes de lo específico del perfil estudiado, las conclusiones no deben leerse como universales, sino como ligadas a unas vivencias específicas como son las de la mujer joven española de una ciudad de tamaño medio.

A continuación, en el Cuadro 1 se detalla la relación de perfiles de las mujeres entrevistadas. ${ }^{6}$

Como se puede observar, el nivel de estudios contempla una muestra diversa: 12 de las 21 entrevistadas $(57,14 \%)$ poseen estudios universitarios ( 7 con los estudios ya acabados) y las 9 restantes tienen un nivel de estudios diverso (Bachillerato o Educación Secundaria). El nivel de estudios se ha aceptado como un factor importante a la hora de estudiar la vinculación con el feminismo (García Jiménez, Cala Carrillo y Trigo Sánchez, 2016). Se opta por perfiles diversos ya que, al tratarse de una investigación centrada en los discursos relacionados con la ciudad, la diversidad de la muestra es importante. 


\begin{tabular}{|c|c|c|c|}
\hline Nombre & Edad & Estudios & Cercanía al feminismo \\
\hline ANA & 25 & Universidad (completo) & Feminista (no colect.) \\
\hline ALBA & 18 & Bachillerato (en curso) & Feminista (no colect.) \\
\hline BEATRIZ & 26 & Universidad (completo) & No concienciada \\
\hline BLANCA & 26 & Universidad (en curso) & Simpatizante \\
\hline CRISTINA & 24 & Universidad (completo) & Simpatizante \\
\hline CLAUDIA & 24 & Universidad (en curso) & Feminista (no colect.) \\
\hline DELIA & 21 & Universidad (en curso) & No concienciada \\
\hline ELISA & 19 & Bachillerato (completo) & Simpatizante \\
\hline FLOR & 21 & Educación Secundaria (completo) & No concienciada \\
\hline GEMMA & 24 & Bachillerato (en curso) & No concienciada \\
\hline ISABEL & 23 & Universidad (completo) & Simpatizante \\
\hline JOANA & 22 & Educación Secundaria (completo) & No concienciada \\
\hline LARA & 24 & Universidad (completo) & Feminista (no colect.) \\
\hline MARTA & 26 & Universidad (completo) & Feminista (colect.) \\
\hline NEREA & 20 & Bachillerato (en curso) & Feminista (colect.) \\
\hline OLGA & 23 & Educación Secundaria (en curso) & Feminista (no colect.) \\
\hline PAULA & 23 & Universidad (completo) & Simpatizante \\
\hline ROCÍO & 20 & Educación Secundaria (completo) & Feminista (no colect.) \\
\hline SANDRA & 23 & Universidad (en curso) & No concienciada \\
\hline TERESA & 25 & Universidad (en curso) & Simpatizante \\
\hline VERÓNICA & 22 & Bachillerato (completo) & Simpatizante \\
\hline
\end{tabular}

En cuanto al nivel de vinculación con el feminismo, hay pocos trabajos en territorio español que estudien los niveles de vinculación cognitiva con el feminismo (ob. cit., 2016). Ante la falta de tipologías canónicas, se propone una tipología maximalista a partir de las entrevistas. En ellas se les preguntó a las mujeres por su nivel de compromiso con el feminismo. Las que negaban cualquier tipo de compromiso por omisión ("nunca me ha interesado") o por rechazo ("demasiado radical", "extremista", "lo mismo que el machismo pero al revés", etc.) son recogidas con la etiqueta "No concienciada" (6 mujeres, el 28,57\%). Las mujeres que presentaban algún tipo de interés (por considerarlo "legítimo" o "necesario") pero que tenían problemas a la hora de autodefinirse como feministas (debido a estereotipos relacionados con esta ideología, como creer que "tampoco son las formas adecuadas", "polarizan demasiado los problemas", etc.) son recogidas con la etiqueta de "Simpatizantes" (7 mujeres, el 33,3\%). Por último, las mujeres que se autodefinían como feministas y que habían integrado parte o la totalidad del discurso feminista son recogidas con la etiqueta "Feministas" (8 mujeres, el 38,1\%; 2 relacionadas con colectivos feministas de Zaragoza) diferenciando entre las que participan en algún colectivo feminista y las que no.

No obstante, las trayectorias personales y la complejidad del pensamiento de cada mujer respecto al feminismo no pueden reducirse a una etiqueta. Existen muchas razones para vincularse o no al feminismo. Para profundizar en los niveles de adhesión a ideas feministas y las
Cuadro 1: Relación de entrevistadas, edad, estudios y nivel de cercanía al feminismo. Fuente: Elaboración propia. 
reticencias para autodefinirse desde este discurso nos remitimos a varios estudios (Williams y Wittig, 1997; Burn, Aboud y Moyles, 2000; Toller, Suter y Trautman, 2004; Leaper y Brown , 2008).

La pregunta de investigación que encardina la investigación es: ¿Existe relación entre la identificación y valoración de las agresiones sufridas y el nivel de vinculación con el feminismo de tal forma que se pueda entender que el feminismo permite poner en marcha procesos de empoderamiento a partir de prácticas de emancipación y enfrentamiento? De esta pregunta se desprenden una serie de hipótesis a contrastar:

- H1.1: Niveles de categoría "Feminista" se relacionan con altas capacidades para identificar las situaciones de violencia y darle un sentido social y colectivo al problema evidenciando su carácter ilegítimo.

- H1.2: Cuando se relaciona la concienciación con la participación en colectivos feministas, además de una capacidad de identificación, hay una creencia en la eficacia de la acción colectiva volcada al cambio.

- H2: Niveles de categoría "Simpatizante" se relacionan con ciertas capacidades de identificación de situaciones de injusticia pero también con relativas incapacidades de dar dimensión colectiva y social a la identificación del problema y la solución del mismo.

- H3: Niveles de categoría "No concienciada" se relacionan con niveles altos de invisibilización de las violencias sufridas (no identificadas como violencia), así como con incapacidad para percibir las situaciones de violencia percibidas como injustas y no legítimas.

Los resultados se estructuran en tres niveles y en cada uno de los niveles se analiza y profundiza en el discurso de uno de los subgrupos, qué relación reconocen con el discurso feminista y cómo este discurso interviene en la forma en la que perciben y actúan en los entornos urbanos. En conjunto, los resultados expuestos arrojarán luz sobre mecanismos de percepción de violencia urbana valiosos por aportar elementos para indagar en las experiencias urbanas de mujeres jóvenes y la manera en la que los discursos de género intervienen en la forma de vivir la ciudad. No obstante, los resultados obtenidos deben ser considerados como específicos del grupo estudiado y sólo podrán ser extrapolados a otros grupos de mujeres cuando investigaciones similares aporten resultados afines.

\section{Resultados}

Las entrevistas partían de la idea de que los procesos de percepción y valoración del entorno no suelen ser ni plenamente conscientes ni accesibles a través de preguntas directas. La falta de costumbre de hablar de este tema haría que una pregunta directa desconcertase al interlocutor. No obstante, esta dificultad se encontró sobre todo en las mujeres más alejadas del feminismo. Las mujeres más o menos cercanas al feminismo presentaron un nivel alto de conciencia respecto a su situación urbana: hablan en términos de acoso callejero, machismo, espacios del miedo, etc. Las mujeres más alejadas del feminismo tardaron más tiempo en entender las preguntas y centrarse en el tema de la experiencia urbana. En ese sentido, varias entrevistas necesitaron un ritmo pausado donde se abordaron los temas a través de preguntas indirec- 
tas: infancia, relación con los vecinos, adolescencia y primeras noches fuera de casa. De esta forma se consiguió crear una situación de confianza donde la entrevistada se encuentra segura en el papel de experta y se relaja al ver la facilidad de las preguntas.

Poco a poco, las preguntas se van dirigiendo hacia las formas en la que la persona entrevistada valora el entorno, las experiencias más significativas en la ciudad, los malos recuerdos y su visión del feminismo. Este tipo de aproximación consiguió establecer un buen clima conversacional, facilitar los testimonios personales y colaborar en la rememoración.

Respecto a las experiencias de las entrevistadas, la totalidad de las mujeres presentaron valoraciones negativas relacionadas con algunos espacios y situaciones similares. Todas habían sufrido distintos niveles de agresiones que iban desde agresiones más "sutiles" como las miradas lascivas, mencionadas por todas las entrevistadas, hasta intentos de violación, relatados por tres de las entrevistadas. La similitud de experiencias sufridas permitirá afinar el análisis de la relación entre nivel de identificación y valoración de las experiencias. Resulta necesario aclarar que, salvo en los casos que se especifique, cuando se habla de agresiones se hace referencia a las agresiones más cotidianas (piropos y vulgaridades, miradas lascivas, silbidos, bloquear el paso para iniciar conversación, etc.) y no tanto a las agresiones más graves como intentos de violación.

\section{El feminismo como factor de visibilización: las "gafas moradas"}

Como se ha dicho más arriba, las mujeres entrevistadas que se identificaban como feministas han mostrado mayor fluidez a la hora de hablar sobre el tema de la experiencia urbana. Esto se muestra en la facilidad para identificar tanto los espacios donde se siente miedo como las razones por las que se siente. Esto se debía a que, a diferencia de las otras entrevistadas, las que se identificaban con el feminismo habían encontrado espacios donde hablar de sus experiencias: grupos feministas, redes sociales, etc. La falta de comunicación respecto a estas experiencias hace que se invisibilicen, lo que refuerza una violencia simbólica que da legitimidad a los contenidos impuestos. Como dice Marta: "si no lo hablas no sabes que es violencia”. Al encontrar formas de expresar las experiencias sufridas, las mujeres feministas pueden verbalizar cómo se sienten y comenzar procesos de empoderamiento.

Para las mujeres que participan en colectivos feministas éstos son muy valiosos por los círculos de confianza que generan. Nerea, por ejemplo, aprecia mucho haber podido "conseguir un espacio de cuidado con tus amigas" ya que "deja hablar estas cosas y ver que todas tenemos mucho en común". Aunque las que no forman parte de un colectivo también han encontrado espacios para hablar (con amigas, como Alba, Claudia y Lara; o con la familia, como Rocío), el colectivo ha significado para Marta y Nerea un verdadero apoyo y espacio de retroalimentación del discurso.

Esto supone un importante elemento de desarrollo de la conciencia crítica necesaria para el empoderamiento (cfr. Gutiérrez, 1995, p. 230): poder identificar áreas de experiencias comunes fomenta la aparición de una "conciencia de grupo". A partir de esta concienciación las mujeres pueden darle un sentido social que contextualiza lo vivido y, así, la mayoría de las mujeres feministas entrevistadas se refieren a 
sus acosadores en términos de "machistas". Como dice Alba: "no, el tío que me acosa no tiene un problema mental, es un acosador de mierda y un machista asqueroso". Esta conciencia crítica es la que permite romper con la legitimidad de los casos de violencia urbana y despertar la indignación. Así, las mujeres feministas presentan un mayor nivel de descontento con los casos sufridos, los cuales reconocen como abusos y no como "cosas que pasan".

Incidiendo en la diferencia entre las mujeres que forman parte de un colectivo y las que no, si seguimos el modelo psicológico de Lorraine Gutiérrez (1995) por el cual el empoderamiento se verá posibilitado a través de las dinámicas de Group Identification, Group Consciousness y Self and Collective Efficacy, en el caso de las mujeres feministas que participan en colectivos parecen cumplirse en general los tres elementos mencionados: existe un sentimiento de conciencia grupal que permite identificar las desigualdades sociales de género y ver el problema socialmente, y esta conciencia de grupo alimenta una identidad de grupo que establece alianzas a través de la empatía y sororidad fruto de experiencias afines. Además, en el caso de las mujeres de colectivos parece haber un sentimiento de eficacia colectiva por el cual se percibe la posibilidad de cambiar las cosas.

Esto último es matizable ya que en las entrevistas no se termina de dejar claro si es una creencia basada en la capacidad de realizar cambios sociales u otro tipo de compromiso, como el identitario. Nerea habla del impacto personal que supone el colectivo por "sentirse arropada". Y a Marta, por su parte, el colectivo le permite "aprender e ir empoderándome con las compañeras e ir haciendo más fuertes las gafas moradas", pero tampoco hace referencias acerca de su fe en la capacidad de cambio real. Sin embargo, sí que recuerda talleres y charlas "de autodefensa", y de "respuestas a las violencias machistas" y reconoce que esos talleres le sirvieron para su día a día. Por lo que la pregunta por la eficacia colectiva podría leerse en estos casos como refuerzo de la self efficacy (Bandura, 1982): la participación en colectivos feministas aportan herramientas discursivas y prácticas para poder modificar las creencias en los cursos de acción que puede llevar a cabo la mujer para lidiar con estas situaciones.

En el caso de las mujeres que se identifican como feministas pero no participan de ningún colectivo, si bien existe una conciencia de grupo social por el cual identifican actitudes como machistas, no hay una identidad sólida de grupo salvo el saberse mujeres de una forma general. La capacidad de identificar el problema como social sí que está presente ya que comentarios como el de Olga reconocen una dimensión social en el acoso: "Yo creo que es dominación (...). Yo creo que esperan que te guste pero que no digas nada. No es una forma de ligar". Sin embargo, la conciencia de grupo social no desemboca en un compromiso político con un colectivo. Ninguna de ellas habla de las razones por las que no se involucran en un colectivo feminista (para profundizar en estas razones, véase: Burn, Aboud y Moyles, 2000 y Toller, Suter y Trautman, 2004) pero sí que hacen comentarios sobre lo positivo del feminismo en varias ocasiones. Incluso se refieren a ellas mismas como feministas, como Ana: "Somos pocas mujeres las que de verdad estamos empoderadas y conscientes. Más feministas de verdad".

Respecto a la eficacia del cambio, la creencia en la eficacia colectiva se remite al grupo de "mujeres" en general: se habla de cambio social, pero en términos culturales amplios ("estamos haciendo historia", "nadie se imaginaba que íbamos a ser tantas", etc.). Esta escala de con- 
ciencia de grupo no logra concretarse en un colectivo de apoyo mutuo, por lo que estas mujeres carecen de las redes de sororidad política de la que hablaban las chicas anteriores. Esto limita los espacios de habla y hará reducir los aprendizajes mediante talleres o charlas. Sin embargo, la eficacia individual percibida sigue siendo alta: estas mujeres creen en el enfrentamiento y la contestación. Alba dice "A mí me molesta que me digan cosas por la calle, y yo contesto a todos". Ana, por su parte, declara: "No pienso en no tener miedo, sino que tengo que enfrentarlo. A mí me molesta mucho pensar que puedo tener miedo. No quiero tener miedo...”.

Sin embargo, mientras Olga cree que "la mayoría, si les dices algo y les contestas, se van", Ana reconoce que su actitud "muchas veces es más de teoría que de práctica". Las situaciones de desigualdad objetivas siguen operando en la relación urbana y, aunque el factor perceptivo sea fundamental, la realidad de la posibilidad de violencia física sigue existiendo. Así, Claudia, aunque suele contestar, respecto a alguna noche en la que le increparon dice: "yo me iba a girar a decirle algo e iba a abrir y a girar, pero mi cabeza dijo "no digas nada, porque este tío se te mete dentro'". Hay, pues, un cálculo racional en el que la contestación no siempre es la solución mejor valorada. El peligro de la agresión física modula la creencia en la eficacia individual.

Frente a unas expectativas de mejora de las condiciones de la mujer fruto del discurso feminista, el choque con las realidades de desigualdad real puede generar un descontento dirigido hacia una misma, como pasa con Olga, que al recordar una vez que subió a casa después de que un hombre forcejase para meterse en su portal, dice: "yo luego no hice nada, y eso me jode. Me jode porque podría haberlo hecho (...) pero no lo hice, en ese momento me sentía tan débil y tan sola... Tan asqueada conmigo misma que no hice nada, me quedé así, en mi cama...". Sin embargo, de este peligro no pueden escapar del todo tampoco las mujeres que participan en colectivos feministas. Nerea, por ejemplo, recuerda cuando un hombre la tocó continuadamente en el autobús y se lamenta: "Una siempre piensa que en esa situación una va a gritarle o a decirle qué haces o algo. Pero yo, que soy muy tirada para delante, me quedé congelada (...). ¡No hice nada!”.

Por lo tanto, la creencia en la eficacia individual, aunque es fundamental para el desarrollo de un empoderamiento efectivo y parece ser eficaz a la hora de visibilizar la violencia y reducir la inseguridad percibida, tiene que lidiar con la realidad de la inseguridad real volviendo más complejo el empoderamiento físico.

\section{Simpatizantes: entre la invisibilización y la frustración}

Las mujeres que no se identificaban como feministas debido a diversos estereotipos sobre el feminismo, se encuentran delante de un dilema. Por un lado, reconocen desigualdades a nivel social referidas a la mujer (aunque muchas niegan la existencia de conceptos como "patriarcado") pero, por otro, tienen muchas dificultades para identificar como derivadas de esas desigualdades las situaciones que viven: los casos de acoso callejero que sufren no serían tanto casos de machismo estructural o relaciones de dominación como casos aislados, aunque reconozcan la existencia de ese machismo dominante.

Esto podría comprenderse desde el concepto de "fatiga de género" que Elisabeth Kelan (2009) aplica a las estructuras organizacionales: mu- 
chas mujeres tienen que lidiar con la deseabilidad de lugares de trabajo igualitarios en entornos de trabajo desiguales en la práctica. Al esforzarse una y otra vez por construir una organización igualitaria, la mujer termina fatigándose y, al final, esta fatiga genera frustración y cansancio. En consecuencia, se terminará generando (de manera involuntaria) filtros de percepción para evitar el malestar causado por dicha disonancia. En nuestras entrevistas, esta teoría podría aplicarse al caso de las simpatizantes ya que demuestran mucho cansancio y malestar por la incapacidad de articular plenamente un discurso feminista que dé sentido a lo vivido y, a la vez, tener ideales de igualdad de género y sufrir desigualdades a nivel urbano. Esta incapacidad de articular una visión feminista viene porque, aunque se realiza una valoración positiva del self efficacy y hay una conciencia de grupo social más o menos desarrollada (depende del caso), hay ciertas incapacidades para reconocerse a sí mismas como parte del grupo desfavorecido y articular un "nosotros-mujeres" pleno.

Esta creencia en la eficacia individual para solucionar las cosas, despegada de la eficacia colectiva y del Group Consciousness, genera una hipertrofia del discurso individualista que es una de las respuestas típicas a la fatiga de género (cfr. Kelan, 2009, p. 205). Aunque haya un reconocimiento de las desigualdades de género, se vive cada situación como un "lo que me pasó es distinto", como dice Teresa.

Existe un dilema entre reconocerse como víctima de unas condiciones objetivas y evitar identificarlas. Las simpatizantes del feminismo parecen estar en una posición fronteriza entre una concienciación de género de corte feminista y la invisibilización de las violencias. Esta doble posición genera un malestar bastante mayor que en el caso de las mujeres feministas entrevistadas: las simpatizantes se expresan varias veces en términos de "cansancio", "frustración" o "tristeza". Como dice Blanca: "El estado de alerta agota mucho. Es agotador el ir a casa, sentir miedo por ver a un hombre". Si bien este agotamiento forma parte de la vida de todas las mujeres, en el caso de las feministas entrevistadas éstas parecen tener una capacidad discursiva que permite culpabilizar al agresor y sentir rabia y frustración social. Las simpatizantes parecen vivir la agresión de una manera más pesimista y angustiante. Resulta muy significativo cómo Teresa reconoce que "(a raíz del acoso constante) construyes un monstruo emocional".

No obstante, lejos de caer en un discurso victimizante, estas mujeres simpatizantes siguen respondiendo a las situaciones de agresión, aunque no dispongan de un marco discursivo colectivo del todo desarrollado. Paula lo dice segura: "yo tampoco me voy a callar. ¿Para qué? ¿Para que siga haciendo esto y no le pase nada? No, prefiero saltarle a ver si así le queda claro". Isabel, por su parte, también reconoce enfrentarse, aunque no esconde su malestar: "Me enfada y me cabrea que me digan esas cosas. (...) He llegado a tener conflictos con hombres en plan 'muérete y déjame en paz'. Y no me marca mucho, pero sí que me molesta. Lo que más me pasa es que me duele y me decepciona”. La mujer simpatizante está obligada a sobrevivir en un entorno poco propicio y sigue siendo en ese sentido un agente urbano que, aunque experimenta la situación de forma más triste, en ningún caso vive la situación de vulnerabilidad de una manera pasiva: gestiona el riesgo y afronta situaciones de inseguridad de la misma manera que las feministas; sólo se diferencia en la capacidad de percibir y defenderse cognitivamente de la agresión. El feminismo, nuevamente, se revela como una pieza importante en el desarrollo de una conciencia crítica que permita a la mujer analizar la situación de violencia y responder a ella con un menor malestar. 


\section{No concienciadas: la invisibilización y la culpa}

Finalmente, nos centraremos en el caso de las mujeres más alejadas al feminismo. De este grupo de entrevistas parece desprenderse una vez más el problema entre, por un lado, conocer (en este caso con menos profundidad) los problemas sociales identificándolos como indeseables y, por otro, no reconocer la violencia sufrida en primera persona como consecuencia de ellos: las mujeres entrevistadas, aunque alejadas del feminismo, reconocen la existencia del machismo, de la violencia de género y los distintos niveles de desigualdad. Sin embargo, tienen un conocimiento superficial de dichos problemas (de las entrevistas se desprenden estereotipos del estilo "hay también mujeres que pegan o manipulan", "tampoco hay que llamar machismo a todo", etc.) y muestran muchas dificultades para reconocer los casos vividos como ejemplos de los mismos. Esto limita fuertemente la capacidad de identificación grupal al ser incapaces de identificar áreas de desigualdad comunes con otras mujeres. En consecuencia, la eficacia colectiva percibida es muy limitada: las entrevistadas hablan constantemente de la dificultad o imposibilidad de solucionar los problemas sociales que les afectan.

Valga como ejemplo Beatriz cuando reconoce que la calle "es la jungla y en la jungla no hay justicia: el más fuerte triunfa”. Asimismo, Sandra dice con pesadumbre: "es lo que hay y tienes que aguantarlo". También Delia dice algo parecido hacia el final de la entrevista: "era como que no hubiese escapatoria. No hay ningún truco para poder evitar esto. Es decir, la violencia va a estar ahí siempre... La voy a vivir de una manera u otra".

Este grupo presenta una incapacidad notable para identificar las agresiones sufridas como parte de unas relaciones de desigualdad indeseables, con lo que se termina desdibujando el carácter colectivo de determinados fenómenos para plantearlos en términos individuales. Esto repercute facilitando que la violencia simbólica normalice la agresión sufrida. Esta normalización opera según dos procesos detectados en las entrevistas: la exculpación y la inculpación.

Por un lado, la exculpación del agresor mediante dos argumentos, según quién agreda: a los agresores conocidos/amigos se les exculpa a través del alcohol y se los cataloga solamente como "un pesado", como hacen Delia, Flor y Gemma. Para Delia, el amigo acosador "es que se pone muy borracho y se vuelve un baboso, pero no es mal tipo", aunque haya habido tocamientos por medio. "Solo quiere ligar, aunque cuando va borracho, le da igual con quién", dice riéndose Flor. Para Gemma, el alcohol es "la diferencia entre un colega y un pervertido".

Cuando el agresor no es conocido se le exculpa igualmente, pero esta vez a través de la figura del "loco" que utilizan Delia, Flor, Joana y Sandra. Esta figura es fruto de la despersonalización total del individuo que agrede: un loco al "que no se le puede enseñar", como dice Joana; un loco que "aparece en cualquier lado", como afirma con miedo Delia, "Si te toca un loco, te toca un loco" dice más adelante hablando de la dificultad de solucionar el problema del acoso callejero. A este loco "se le puede ir la cabeza y hacernos de todo", por lo que se trata de un monstruo urbano que alimenta el miedo y la inseguridad pero que no se relaciona con un problema social como es el machismo o la desigualdad entre géneros: el loco es otra forma de ignorar la relación entre las violencias sufridas y la estructura social. 
El otro proceso de normalización de la violencia es el de la inculpación de la agredida: Gemma, Joana y Sandra hablan de sí mismas en términos de "tontas" ya que, como dice Gemma, "también es que me meto en cada fregado...." “Quién me manda a irme con ese tipo?”, se lamenta Joana cuando cuenta un intento de violación. Gemma recuerda lo que le dijo su amiga cuando salieron de una situación de acoso muy grave "'Chicas, esto nos sirve para aprender para la próxima', me dijo mi colega, y la verdad es que tiene razón...”. Para Sandra, ella "les llama” por cómo viste o por "darles coba" hablando.

Esta inculpación pone la responsabilidad en una misma generando frustración: "yo es que soy tonta" dijeron Joana y Sandra. Beatriz, por su parte, condensa todo en una frase: "si una se echa la culpa a sí misma como que es menos doloroso. Mi cabeza, para que no me duela, me dice que me lo he buscado "cómo no va a pasar, es normal'".

Sin embargo, volviendo a plantear a la mujer no como víctima sino como agente, en estas entrevistas se conjuga la incapacidad de ver el componente colectivo de las agresiones con una creencia bastante fuerte en la autoeficacia en términos tácticos: se sabe que las agresiones seguirán existiendo y se toma la situación de forma pesimista pero, lejos de verse inhabilitadas para una respuesta, se desarrolla una inteligencia pragmática volcada en intentar salir de cada situación de manera calculadora. En las entrevistas de las mujeres no concienciadas sale a relucir menos descontento y frustración respecto a las agresiones sufridas, posiblemente por no tener muchas expectativas en un orden distinto de las cosas.

Los métodos de solución de conflictos son en estas mujeres menos contestatarios y más dialogantes. Joana dice sobre un hombre que le empezó a seguir por la noche: "yo le seguí el rollo sonriéndole porque no quise mostrarle miedo siendo simpática para que no se asustara, no se alterara y no empleara otra estrategia...”. Beatriz, respecto a la necesidad de solucionar in situ las situaciones de acoso, vuelve a expresarse de manera sorprendente y dice: "Al final la dignidad va detrás de tu vida. Prefiero hacerme la tonta antes de que me peguen una paliza o me violen".

A través de estas formas de solución de problemas se podría entrever una especie de cinismo operativo centrado en la supervivencia individual a cualquier precio, aun incluso a costa de la dignidad y el autoconcepto ("yo me siento muy mal y muy ridícula haciendo estas cosas, pero ¿qué quieres?”, dice Joana). Como dice Gemma con pesimismo: "al final una hace lo que puede. Se trata al final de sobrevivir. Pero qué triste. Y saber que siempre que salgas te va a pasar algo...”.

\section{Conclusiones}

El objetivo de este artículo ha sido demostrar la utilidad que tiene un discurso feminista como herramienta de empoderamiento necesaria para la innovación social al colaborar en la visibilización y confrontación de las agresiones urbanas. Se profundizó en la forma en que el discurso feminista permitía a las mujeres dar sentido a las agresiones sufridas y poner en marcha procesos de solución colectiva. De las hipótesis que se plantearon, la mayoría se han visto apoyadas por los resultados, aunque varias con matizaciones.

La hipótesis 1.1 se ha visto apoyada por los resultados al mostrarse en las entrevistas cómo el feminismo permite articular un discurso que 
permite resignificar situaciones vividas como parte de problemas sociales y plantearlos como ilegítimos. No obstante, hay que lidiar con el conflicto que supone tener unas expectativas de mejor social y el hecho de vivir situaciones de agresión constantes. La hipótesis 1.2 también es apoyada por los resultados al ser las mujeres en colectivos feministas las que tienen mayor percepción de cambio posible (aunque no se ha constatado si es una creencia en cambio real o cambio cotidiano en la vida de las del grupo).

La hipótesis 2 se apoya parcialmente en los resultados al haber efectivamente limitaciones a la hora de identificar los casos sufridos como consecuencias de problemas sociales (debido posiblemente a la "fatiga de género"). Sin embargo, sí que hay creencias de igualdad y eficacia colectiva aunque de una manera amplia y abstracta.

La hipótesis 3 no se ha visto del todo apoyada ya que sí que parece haber una capacidad relativa de identificación de las situaciones de desigualdad sociales, aunque la relación de dicha situación con la propia vida es muy débil ("no es mi caso"). La desconexión de los casos sufridos con la realidad social se da a través de la exculpación del agresor y de la inculpación de la víctima. No obstante, aun en situación de agresión la mujer no se ve inhabilitada para la acción, sino que responde de una forma pragmática, incluso a costa de su dignidad.

Estas matizaciones demuestran que el proceso de tejer una experiencia en entornos de desigualdad se muestra siempre como dinámico y complejo: la tensión permanente entre la vulnerabilidad y la resistencia es incorporada de maneras muy diversas, dando lugar a formas de relacionarse con el entorno sumamente plurales.

Integrar en las políticas urbanas el enfoque feminista al reconocer en el feminismo una herramienta de visibilización de conflictos y de empoderamiento de las poblaciones en situación de vulnerabilidad (aunque no pasivas ni inhabilitadas como agentes) resulta importante para poner en marcha procesos de innovación social. La ciudadanía se pondrá manos a la obra en la mejora social y urbana si es capaz de identificar situaciones de violencia y de acceder a la reflexión de las mismas en pos de una mejor ciudad para todas. 


\section{Referencias}

Alexander, A. y Welzel, C. (2011). Empowering Women: The Role of Emancipative Beliefs. European Sociological Review, 27(3), 364-384.

Añover López, M. (2012). Los espacios “del miedo”, ciudad y género. Experiencias y percepciones en Zaragoza. Geographicalia, 61, 25-45.

Balcázar Nava, P., González-Arratia, N. I., Gurrola Peña, G. M. et al. (2006). Investigación cualitativa. Ciudad de México: Universidad Autónoma del Estado de México.

Bandura, A. (1982). Self-efficacy mechanism in human agency. American psychologist, 37(2), 122-147.

Berguer, P.L. (1989). Introducción a la sociología: Una perspectiva humanistica. México: Limusa.

Blanco, I. y Subirats, J. (2012) . Políticas urbanas en España: dinámicas de transformación y retos ante la crisis. Geopolitica(s), 3(1), 15-33.

Bourdieu, P. (2000). La dominación masculina. Barcelona: Anagrama.

Bridge, G. y Watson, S. (2000). City Publics. A companion to the city. Oxford: Blackwell Publishers.

Burn, S., Aboud, R. y Moyles, C. (2000). The relationship between gender social identity and support for feminism. Sex Roles, 42(11-12), 1081-1089.

Casey, R., Goudie, R. y Reeve, K. (2008). Homeless Women in Public Spaces: Strategies of Resistance. Housing Studies, 23(6), 899-916.

Chiappero-Martinetti, E., Houghton Budd, C. y Ziegler, R. (2017). Social Innovation and the Capability Approach - Introduction to the Special Issue. Journal of Human Development and Capabilities, 18(2), 141-147.

Ciocoletto, A. (2014). Espais per a la vida quotidiana. Auditoria de qualitat urbana amb perspectiva de gènere. Barcelona: Col.lectiu Punt 6.

Ciocoletto, A., Gutiérrez Valdivia, B. y Ortiz Escalante, S. (2014, febrero). Vivir en áreas residenciales monofuncionales desde la perspectiva de género. En I Congreso Internacional de Vivienda Colectiva Sostenible. Barcelona, 25, 26 y 27 de febrero de 2014. Barcelona: Máster Laboratorio de la Vivienda Sostenible del Siglo XXI, 286-291.

Coffey, A. (1995). Dones i urbanisme. Àrea. Revista de Debats Territorials, 3, 4-22.

Cresswell, T. (2010). Towards a Politics of Mobility. Environment and planning D: Society and space, 28(1), 17-31.

Delgado, L. S. y Aguerri, J. C. (2015) Más allá del miedo urbano. Prácticas de resignificación espacial y supervivencia a la violencia en la ciudad de Zaragoza. Encrucijadas: Revista Crítica de Ciencias Sociales, 15, a1502. Recuperado el 30 de julio de 2018, de: http:/ / www.encrucijadas.org/index.php/ojs/article/view/348/273.
Díaz, M. (1989). Movilidad femenina en la ciudad. Notas a partir de un caso. Documents d'analisi geográfica, $14,219-239$.

Díez Jorge, M.E. (ed.) (2015). Arquitectura y mujeres en la historia. Madrid: Síntesis.

Doan, P. (2007). Queers in the American City: Transgendered perceptions of urban space. Gender, Place and Culture, 14(1), 57-74.

Durán Heras, M. (2017). La ciudad compartida: urbanismo y movimientos sociales. En Serrano Lanzarote, B., Mateo Cecilia, C. y Rubio Garrido, A. (eds.), Género y política urbana. Arquitectura y urbanismo desde la perspectiva de género. Valencia: Generalitat Valenciana, 33-58.

Ehrkamp, P. (2013). 'I've had it with them!' Younger migrant women's spatial practices of conformity and resistance. Gender, Place E Culture, 20(1), 19-36.

Fairchild, K. y Rudman, L. (2008). Everyday Stranger Harassment and Women's Objectification. Social Justice Research, 21(3), 338-357.

García Jiménez, M., Cala Carrillo, M. y Trigo Sánchez, M. (2016). Conocimiento y actitudes hacia el feminismo. Femeris, 1(1-2), 95-112.

Glaser, B. y Strauss, A. (1967). The discovery of grounded theory. Chicago: Aldine.

González, S., Moulaert, F. y Martinelli, F. (2010). ALMOIN: how to analyse social innovation at the local level? En Swyngedouw, E., Martinelli, F., Gonzalez, S. et al. (eds.), Can Neighbourhoods Save the City? Community Development and Social Innovation. London: Routledge, 49-67.

Greed, C. (1996). Promise or progress: women and planning. Built Environment, 22(1), 9-21.

Gutiérrez, L. (1995). Understanding the empowerment process: Does consciousness make a difference? Social Work Research, 19(4), 229-237.

Ibañez, J. (1997). Más allá de la sociología. El grupo de discusión: Técnica y crítica. Madrid: Siglo XXI.

Kabeer, N. (1999). Resources, Agency, Achievements: Reflections on the Measurement of Women's Empowerment. Development and Change, 30, 435-464. DOI: https://doi.org/10.1111/1467-7660.00125.

Kelan, E. (2009). Gender fatigue: The ideological dilemma of gender neutrality and discrimination in organizations. Canadian Journal of Administrative Sciences, 26(3), 197-210.

Leaper, C. y Brown, C. (2008). Perceived experiences with sexism among adolescent girls. Child Development, 79(3), 685-704.

MacMillan, R., Nierobisz, A. y Welsh, S. (2000). Experiencing the Streets: Harassment and Perceptions of Safety among Women. Journal of Research in Crime and Delinquency, 37(3), 306-322. 
Magrinyá, F., \& de Balanzó, R. (2015). Innovación social, innovación urbana y resiliencia desde una perspectiva crítica: el caso de la autoorganización en el espacio urbano de Barcelona. En Subirats, J. y García Bernardos, A. (eds.), Innovación social y politicas urbanas en España. Experiencias significativas en las grandes ciudades. Barcelona: Icaria Editorial, 59-93.

McCall, L. (2005). The Complexity of Intersectionality. Signs: Journal of Women in Culture and Society, 30(3), 1771-1800.

Miles, M. y Huberman, M. (1994). Qualitative data analysis: An expanded sourcebook. Thousand Oak: Sage.

Miralles-Guasch, C. (1998). La movilidad de las mujeres en la ciudad, un análisis desde la ecología urbana. Ecología Política, 15, 123-131.

Moulaert, F., MacCallum, D., Mehmood, A., et al. (2013). General introduction: the return of social innovation as as scientific concept and a social practice. En Moulaert, F., MacCallum, D., Mehmood, A., et al. (eds.), The international Handbook on Social Innovation: Collective Action, Social Learning and Transdisciplinary Research. Cheltenham: Edward Elgar, 1-6.

Muxí, Z., Casanovas, R., Ciocoletto, A., et al. (2011). ¿Qué aporta la perspectiva de género al urbanismo? Feminismo/s, 17, 131-153.

Olmo Sánchez, M. y Maeso González, E. (2012). Diferencias de género en la movilidad en regiones urbanas de Andalucía. En Investigación y género, inseparables en el presente y en el futuro: IV Congreso Universitario Nacional Investigación y Género. Sevilla: Facultad de Ciencias del Trabajo de la Universidad de Sevilla, 1353-1372.

Ortiz Escalante, S. (2017). El lado nocturno de la vida cotidiana: un análisis feminista de la planificación urbana nocturna. Kultur: revista interdisciplinaria sobre la cultura de la ciutat, 4(7), 55-77. DOI: http:/ / dx.doi.org/10.6035/Kult-ur.2017.4.7.2.

Ortiz i Guitart, A. (2007). Hacia una ciudad no sexista. Algunas reflexiones a partir de la geografía humana feminista para la planeación del espacio urbano. Territorios, 16-17, 11-28.

Pain, R. (2001). Gender, Race, Age and Fear in the City. Urban Studies, 38(5-6), 899-913.

Panelli, R. (2005). Claiming space and communit: rural women's strategies for living with, and beyond, fear. Geoforum, 36(4), 495-508.
Pradel Miquel, M. y García Cabeza, M. (2018). Innovación social en las ciudades españolas: la centralidad de la gobernanza local y ciudadana. En Pradel Miquel, M. y García Cabeza, M. (eds.), El momento de la ciudadanía. Innovación social y gobernanza urbana. Madrid: Catarata, 13-31.

Rodó-de-Zárate, M. (2011). El jovent i els espais públics usbans des de la perspectiva de gènere. Un estat de la qüestió des de la geografía. Documents D’Analisi Geografica, 57(1), 147-162.

Rodó-de-Zárate, M. (2015). Young lesbians negotiating public space in Manresa: an intersectional approach through places. Children's Geographies, 13(4), 413-434.

Sánchez de Madariaga, I. (2004). Infraestructuras para la vida cotidiana y calidad de vida. Ciudades, 8, 101133.

Sandercock, L. y Forsyth, A. (1992). A gender agenda: new directions for Planning Theory. American Planning Association Journal, 58, 49-59.

Sen, A. (1984). Resources, Values and Development. Oxford: Blackwell.

Sen, A. (1985). Commodities and Capabilities. Amsterdam: North-Holland.

Sime, D. (2017). Migrant Children in Cities: The Spatial Constructions of Their Everyday Lives. En Ni Laoire, C., White, A. y Skelton, T., Movement, Mobilities, and Journeys. Geographies of Children and Youg People. Singapore: Springer, pp. 271-288.

Toller, P., Suter, E. y Trautman, T. (2004). Gender Role Identity and Attitudes Toward Feminism. Sex Roles, 51(1-2), 85-90.

Van Lieshout, M. y Aarts, N. (2008). Outside is where it's at! Youth and immigrants'perspectives on public spaces. Space and Culture, 4(12), 497-513.

Vidal Moranta, T. y Pol Urrutia, E. (2005). La apropiación del espacio: una propuesta teórica para comprender la vinculación entre las personas y los lugares. Anuario de Psicología, 36(3), 281-297.

Vinuesa Angulo, J. (2000). Los jóvenes en la estructura demográfica de la región metropolitana de Madrid. Estudios Geográficos, 241, 683-715.

Williams, R. y Wittig, M. (1997). 'I'm not a feminist, but...': Factors contributing to the discrepancy between pro-feminist orientation and feminist social identity. Sex Roles, 37(11-12), 885-904. 
Delgado, L.S. (2018). La utilidad del feminismo. Empoderamiento y visibilización de la violencia urbana en las mujeres jóvenes. Hábitat y Sociedad, 11, 131-148.

<http://dx.doi.org/10.12795/HabitatySociedad.2018.i11.08>

$\bigcirc$ 